\title{
On Information Modeling to Support Interoperable Spatial Databases
}

\author{
Nectaria Tryfona \\ National Center for Geographic Information and Analysis \\ Boardman Hall, University of Maine, Orono, ME 04469-5711, U.S.A. \\ nectaria@spatial.maine.edu \\ and \\ Jayant Sharma \\ National Center for Geographic Information and Analysis \\ Department of Spatial Information Science and Engineering \\ Boardman Hall, University of Maine, Orono, ME 04469-5711, U.S.A. \\ jayant@spatial.maine.edu
}

\begin{abstract}
A major research topic in geographic databases is that of interoperability, i.e., the capability of integrating transparently remote data and processes in an open environment. This paper addresses the issue of information modeling for interoperating geographic databases. In particular, it deals with the topic of exchanging semantics at the information systems context. Our proposal is based on the peculiarities of spatial data, namely field- and object-based views of space and spatial relationships, that are critical for the representation of information in an interoperable, yet unified "system". A generic Geographic Data Model that encapsulates these semantics and makes their interchange among remote systems possible and without ambiguities is discussed. We show, with an example, how this model supports interaction among heterogeneous spatial application domains. This research effort is based on (a) the requirements expressed by the OpenGIS community, (b) results from modeling "classical" interoperable applications, and (c) a well-established theory on database modeling of geographic applications.
\end{abstract}

\section{Introduction}

Geographic Information Systems (GIS) are decision support tools based on the collection, storage, retrieval and representation of spatial data. An Interoperable (or Interoperating) GIS is one that allows remote systems seamless and transparent access to its functionality and data. It is a distributed yet unified environment, in which all supported models and processes are compatible. The heterogeneous remote systems are autonomous and "logically" connected providing meaningful exchange of data (Goh et. al., 1994). In a multisystem GIS all components conform to generic data and process models, regardless who produced them, how, when and why. The difference between an interoperable and a distributed environment is that, in the former, various applications share and exchange processes and data, while in the latter all processes and data belong to the same application.

Interoperability of GIS is currently a central research and development effort for the geographic data processing community. It is fueled by the growing need for developing applications based on interaction among various hardware and software components located at different sites, owned by different "vendors", and designed for widely differing application domains. It is also observed that current technology is difficult to use when multiple systems are invited to work together, each one independently, forming an integrated environment. 
Therefore the range of issues to be dealt in interoperable spatial information systems is quite broad. Starting with designing open architectures that allow access to geographic information on distributed, heterogeneous systems and ranging till the development of data and process models suitable for these open architectures, a requirements analysis for spatial operations, study of the properties of space that contribute to peculiarities in spatial data handling, and specification of complex interdependencies and consistency constraints between geographic data and operations.

Interoperability is not a new concept. It has been around for many years, primarily in the computer science research community, for describing solutions to build systems by combining heterogeneous resources and services (Manola et. al., 1992; Soley, 1992; Elmagarmid and $\mathrm{Pu}, 1990$ ). Although interoperability of systems is a major research focus for various domains, (see for example, heterogeneous databases (Sheth and Karabaitis, 1993; Sciore et. al., 1994; Sheth and Kalinichenco, 1992; Kashyap and Sheth, 1994), medical information systems (Mannal and Burgara, 1993), and business applications (Chen et. al., 1993)), each one has its own peculiarities that must be addressed. Common to all domains, however, are the issues of cooperation, and capturing and exchanging semantics among multiple databases and applications. There are several approaches to these aspects: Schek and Wolf (1992) describe mechanisms for the cooperation between object database systems and autonomous operation services in a heterogeneous environment. Their architectural framework for interoperability between database management and application-specific computation is based on a kernel model which exists on top a generic data model used by all components. DeLorenzi and Wolf (1993) propose a protocol for Cooperative Spatial Information Managers linking several software components of an open GIS, that is, statistics packages, computational geometric algorithms, record storage managers, spatial storage managers and users' applications. Goh et. al. (1994) address the issue of context interchange in a dynamic environment such as a stock market. Their work tackles more than just schematic and semantic incompatibilities among interoperating systems by providing a mechanisms for expressing and exchanging context. Despite the varied approaches and issues tackled all of the above research efforts assume the existence of a generic data model known to all remote systems.

This paper deals with the issue of a generic spatial data model suitable for facilitating interoperability of GIS. In other words, we address the topic of information modeling for interoperable spatial databases. Spatial databases are an indispensable part of GISs and in an interoperating environment their role is to support the exchange of data and operations as well as the semantics of geographic information. This work examines the special semantics of spatial data in an information systems context and describes a geographic model to encapsulate the distinguishing properties of space. The major contribution of this paper is: (a) the study and definition of special issues which arise in spatial databases that any satisfactory model must handle for supporting interoperability among autonomous and heterogeneous applications, and (b) the presentation of a formal model which can serve as the intermediate model understandable by all the remote systems comprising the spatial interoperating environment.

Our current work is based on: (a) the requirements specifically expressed by the Technical Committee of the Open Geodata Interoperability Specification (OGIS, 1995), (b) results from studying interoperability among GISs (Voisard and Schweppe, 1994), (c) research results from modeling "classical" applications in an interoperating environment (Schek and Wolf, 1992; DeLorenzi and Wolf, 1993; Kim, 1995; Goh et. 
al., 1994), and (d) modeling issues of spatial databases (Hadzilacos and Tryfona, 1992; Delis et. al., 1994; Tryfona, 1994; Tryfona and Hadzilacos, 1995).

The remainder of the paper is organized as follows: Section 2 gives the conceptual framework of an open environment, presents an overview of levels of an interoperable architecture, and focuses on a specific proposal for interoperable geographic databases. Section 3 describes the semantics of spatial information which must form the basis of a successful model for interoperable databases, while Section 4 presents a generic model for supporting and facilitating interoperability, and proposes its use as the enterprise model for cooperating spatial applications. Section 5 gives an example of the model use and Section 6 concludes with a summary of the results and a discussion of future work.

\section{The Conceptual Framework}

Specific results from all aforementioned efforts can be used to accomplish an open GIS environment. On the other hand, the complexity of an interoperable GIS leads to the idea of layer decomposition (Voisard and Schweppe, 1994). Each layer corresponds to a different level of abstraction, starting with the application or user level down to the invocation of system services. There are four basic layers: (a) the application layer, which is concerned with application depended user requests, (b) the abstract services layer, which is a uniform view of the overall system (c) the concrete services layer, which has a view of precise operations that can be asked of each system, and (d) the system services layer, which deals with invocation of services to the specialized system (Voisard and Schweppe, 1994) (Figure 1).

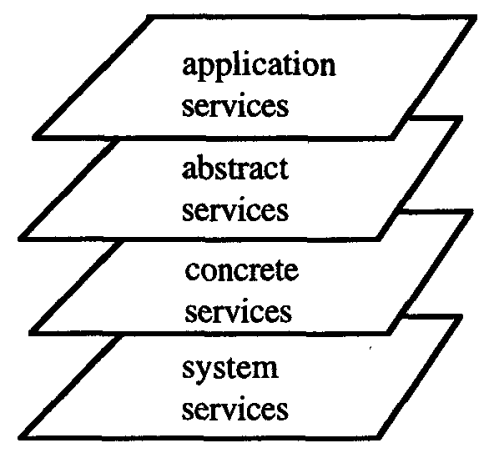

Fig. 1. The layered architecture for an interoperable environment proposed by (Voisard and Schweppe, 1994).

From the perspective of data modeling, the first layer (i.e., application services) deals with user views, while the second one (i.e., abstract services) addresses the issue of definitions of objects, properties, relationships and operations among them. At these two levels, in an interoperable environment, there is a need to exchange data which correspond to portions of applications and hence databases. Exchanging just computer metaphors, e.g., records, layers and processes among the remote systems, leads to lack of data semantics (Hadzilacos, 1995). Semantics in GISs have a special role since the same information can be represented in different ways and data with similar 
representations may have totally different meaning (Abel and Kilby, 1994; Pascoe and Penny, 1995), (Hadzilacos, 1995).

We base our proposal on the layered approach of (Voisard and Schweppe, 1994). In particular, we address the issue of exchanging semantics and data by using a Geographic Data Model (GDM). Figure 2 illustrates the rationale of interoperable geographic databases from the database modeling viewpoint. Each remote system provides an interface for the spatial application built on top of a GIS. Each application communicates with the local data by using a local DataBase Management System (DBMS) and with remote data and processes through the GISs. The GISs communicate via GDM by providing a mechanism to translate their particular model to GDM and vice versa.

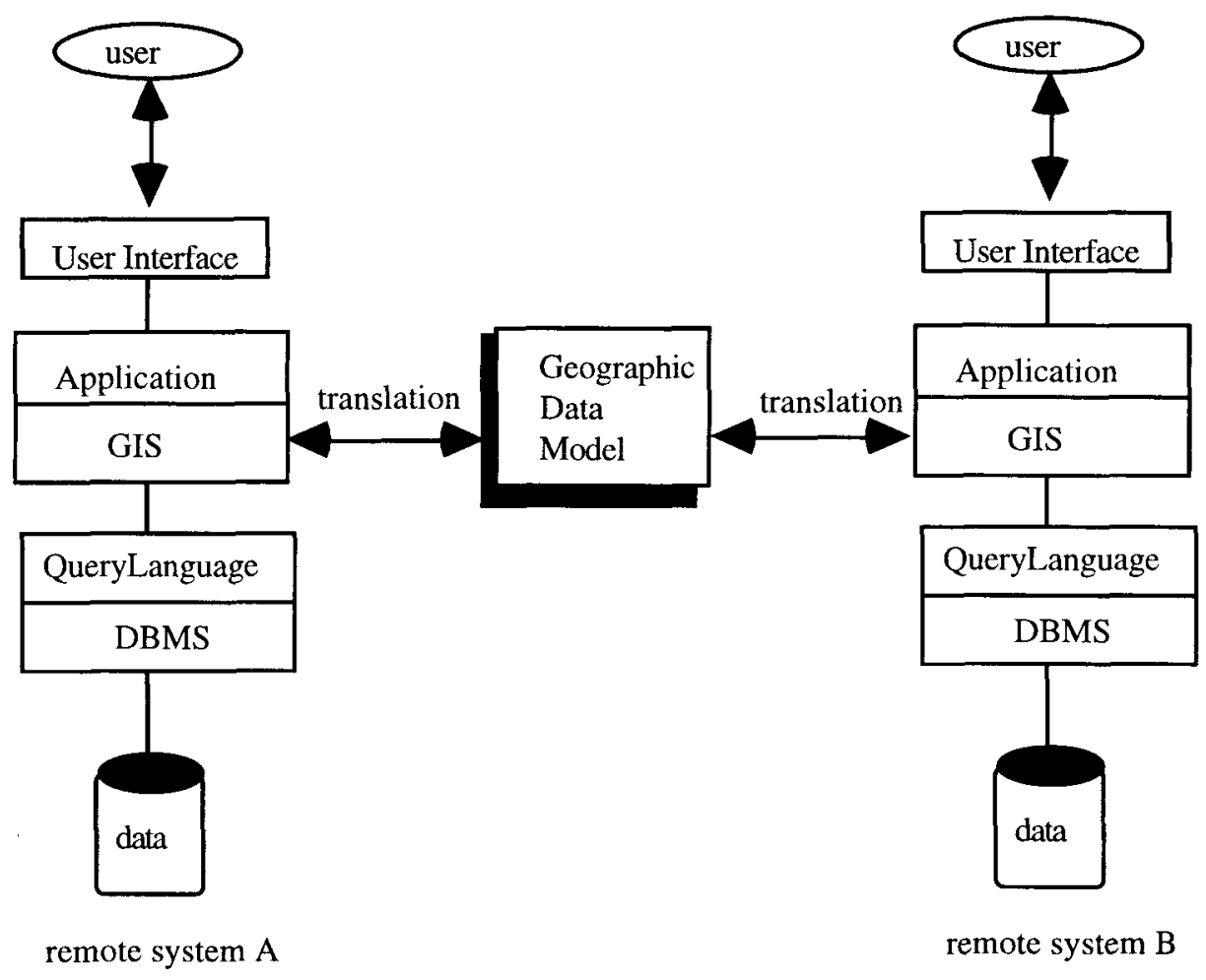

Fig. 2. Interoperating Geographic Databases.

GDM acts as an abstract level communication protocol among the remote systems. It is responsible for representing (a) user views, and (b) objects and operations, formally, without ambiguities, in a way understandable, adaptable and transferable to models that remote systems use. It includes data definition facilities to homogenize different representations of semantically equivalent data in remote systems at different levels of detail. Therefore, GDM corresponds to the application and abstract services layers of Figure 1. 


\section{Semantics of Spatial Information and Desiderata for Modeling}

A clear understanding of the semantics of spatial information will facilitate data modeling for interoperable spatial databases. The what and how of data semantics, its representation, and exchange is the topic of much discussion. We adapt Sheth's proposal (Sheth, 1995) that:

(a) there is a difference between semantics captured by human beings and computers (we deal with the latter),

(b) in the information systems context, semantics can be viewed as a mapping between an object of the real world and one modeled, represented and/or stored in an information system,

(c) the process of capturing semantics by a computer can be improved by defining the syntax of the data, and

(d) regularities in databases (i.e. constraints) that capture objects' behavior in the realworld are components of the semantics.

We also concern ourselves with the semantics of spatial information as a whole, rather than the semantics of individual spatial entities, since all entities inherit these common properties and behavior (Tryfona and Hadzilacos, 1995).

Based on experience from specific application development (UtilNets, 1994) as well as from general studies (Couclelis, 1992), (Camara et. al., 1992), we came to the result that the modeling framework for the semantics must at least handle:

- the field- and object-based views of geographic space, and

- spatial relationships.

We analyze these concepts and then we show why they are critical for the representation of spatial information in an interoperable environment:

- The domain expert often characterizes geographic objects by using spatial varying properties, such as the soil_type of a land_parcel, or the height of a mountain (Tryfona and Hadzilacos, 1995). In fact these are properties of the space inherited by the geographic object of interest since if one object was replaced by another without changing the spatial location, these properties would remain the same. For example, if two land parcels are combined to form a new one then the soil_type of the combined parcel is that of its predecessors, whereas if the boundaries of a land_parcel change, the soil_type may also change (Tryfona and Hadzilacos, 1995). In general, if several objects occupy the same space then they share the same values for their space-varying properties. Therefore, it would be more accurate to assign these properties to "space" (as fields) and provide a mechanism for objects to "inherit" properties of space at the occupied position (Tryfona and Hadzilacos, 1995). The orthogonal object and field views of space are rather deep issues with counterparts in physics and philosophy as atomic and plenum views (Couclelis, 1992). The dichotomy is most likely based on human cognition which "appears to make use of both the object and field views, but at different geographic scales and for different purposes" (Couclelis, 1992). For modeling of geographic applications designers need both field and object views at their disposal. That is, geographic objects can be discrete entities with boundaries separating them (the requirement on handling fuzzy boundaries notwithstanding), however it must also be possible to describe spatial properties, such as ground_elevation and vegetation, without attributing them to specific objects. 
- Describing relationships among objects is the key of capturing semantics (Sheth, 1995). A major issue in geographic databases is expressing spatial relationships among geographic entities. Spatial relationships not only express interdependencies among objects but also integrity constraints on a collection of related objects. For example, the spatial relationships $\mathrm{A}$ inside $\mathrm{B}$ and $\mathrm{B}$ inside $\mathrm{C}$ imply the relationship $\mathrm{A}$ inside $\mathrm{C}$. Any generic model should be able to express spatial integrity constraints, define geographic object classes determined through spatial relationships, and express spatial queries. The definition of a Square, in a cadastral application, as "a land parcel which is not contained in any building block" is an example of using topological integrity constraints. The models should also lead to straightforward solutions for explicitly storing topology in the logical and physical levels -a common practice despite topology being derivable from object positions (Hadzilacos and Tryfona, 1992).

The above concepts and requirements are a consequence of the nature of the domain -spatial in this case- rather than individual objects. Therefore a generic model which will make possible the interchange of spatial information in an interoperable environment must encapsulate the special semantics of this domain, as well as permit the standard syntactic and semantic definitions of entities required for an open information system.

\section{The Geographic Data Model}

This section discusses the Geographic Data Model (GDM) which can serve as the enterprise model of the interoperable environment. GDM captures the semantics of a geographic database, i.e., the concepts described in the previous section. We don't invite others at remote locations to use our model. Each remote system (application) (Figure 2) may use a different model and language. As they express the same aspects, all models are just different syntactic versions of the same underlying spatial concepts encapsulated by GDM; hence, they can be used interchangeably.

The following presentation of GDM is based on Hadzilacos and Tryfona's (1992; 1994) earlier work which uses an object-oriented approach and an unambiguous syntax. We first describe how GDM deals with geographic objects, fields, operations among them, and spatial relationships and integrity constraints. Next we present the mechanism of GDM to accommodate the object-based approach $(\$ 4.1)$, relationships among objects $(\$ 4.2)$, the field-based approach (the concept of layers and operations among them, \$4.3) and the interrelationship between the object and field approaches $(\$ 5)$.

\subsection{Dealing with Geographic Objects}

A database is a set of objects which represent part of the real world. Each object belongs to an object class (or entity set) characterized by a set of properties or attributes, and a set of methods or operations. Each attribute is associated with a domain, which is an unrestricted set of values. Methods are the only means to access the attributes. So each object instance (entity) in a database is represented by a set of values each belonging to the domain of the corresponding attribute of the object class.

In geographic applications spatial objects have a position which links the object with space. Position is a function defined on geographic objects and for each object it returns a part of space (Tryfona and Hadzilacos, 1995). This approach places no restriction on the model of space. For example, 2-dimensional Euclidean space is modeled using an entity set homomorphic to $R^{2}$, i.e., the space contains sets of points. 
The entity set homomorphic to $R^{2}$ corresponds to vector systems, while the one homomorphic to $Z^{2}$ corresponds to raster systems.

Objects in geographic databases have another special attribute, namely their dimension. Its domain is $0,1,2$, or null, and corresponds to the geometric types point, arc, and region. Objects with a null dimension are aspatial (or non-geographic) while non-null dimension objects are called geometric or geographic objects and have a position (Hadzilacos and Tryfona, 1992).

Geometric operations are performed on objects' positions. The primary geometric operations are assumed to be defined together with a domain. Strictly speaking these operations are defined on objects and not on their positions. It simplifies the statement of the definitions, however, and creates no confusion if we describe them as acting on positions (see (Hadzilacos and Tryfona, 1992; Delis et. al., 1994) for the formal treatment, on which the following set of primary geometric operations is based). The set of operations includes: (a) primary operations whose range is real, like distance and area, (b) primary operations whose range is a geometric type, like union, difference, nodes, etc., (c) derived operations whose range is real, like perimeter and length, and (d) derived operations whose range is a geometric data type, like intersect.

The above operations on objects are concerned with the attribute position and hence with spatial relationships among the objects. The next section describes the facilities within the GDM for expressing spatial relationships and constraints.

\subsection{Dealing with Spatial Relationships}

A relationship is a condition on a tuple of values of objects' attributes. Relationships which are conditions on the attribute position are called spatial. In geographic applications topological relationships are a critical subset of the various kinds of spatial relationships. An accepted and commonly used definition of topological relationships between two geographic objects is based on point-set topology (Egenhofer and Herring, 1991). Topological relationships between geometric objects are characterized by considering empty and non-empty intersection of their boundaries and interiors and are called elementary topological relationships.

In GDM, complex topological relationships, integrity constraints, and queries, are constructed using predicate calculus expressions. A topological sentence is built out of atomic topological formulae with negation, conjunction, disjunction, and universal and existential quantification (Hadzilacos and Tryfona, 1992).

Based on the above, the example of Section 3: "A square is a land parcel that is not contained in any building block" is transformed into:

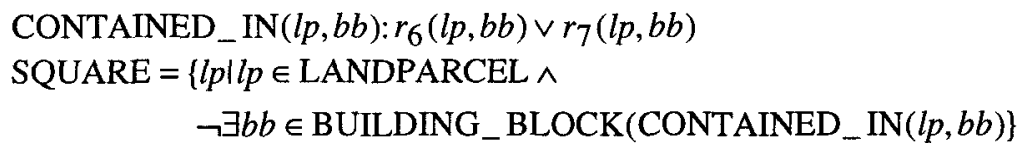

Thus objects can be interrelated in a geographic database and participate in complex spatial integrity constraints and queries.

\subsection{Dealing with Layers (fields)}

A fundamental requirement of spatial database design is the ability to model spatial properties, i.e., to associate parts of space with an attribute. Spatial applications deal 
with the two orthogonal generalizations of spatial properties (Hadzilacos and Tryfona, 1994): (a) the association of the whole space with one attribute (the field view), and (b) the associations of sets of attributes with a geometric figure (the object view). The first is modeled with concepts such as layers, whereas the second is modeled with concepts oriented towards objects. This section describes how the field view is supported by GDM.

Dealing with spatial varying attributes means dealing with layers. A layer is a set of geometric figures (which are representations of geographic objects, consisting of a boundary and an interior) with associated values. Thus, it is natural to define a layer as a function from geometric figures to attributes or as a relation with the geometric figure as the key attribute (Delis et. al., 1994). From the user's point of view layers are often represent derived information. In such cases they are called virtual and they are related with the way users need to view data -a concept similar with that of database views. In manipulating layers it is sufficient to be able to modify the geometric figures (i.e. the domain of the function) or the attributes (i.e. the range of the function) and to combine such changes through function composition. There are with four types of operations forming the functional algebra (Delis et. al., 1994):

(a) Operations (COMPUTE ATTRS) on a single layer function, which change its range (by adding, change or delete non-geometric attributes), but leave the same domain, i.e., the same geometric figures. Such operations are used to derive computable attributes.

(b) Operations (COMPUTE SPATIAL) which operate geometrically on the domain of a layer function to produce a layer function with a new set of geometric figures as domain. For example: given a layer with lines and regions (representing rivers and lakes) construct a layer which consists of a buffering zone around the rivers and lakes.

(c) Reclassification (RECLASS), which operates on a single layer and concatenates adjacent figure if their range, i.e., non-geometric attributes, are identical.

(d) Overlay (OVERLAY), which takes two layer functions and produces a new one with the geometric overlay as domain and the combination of the ranges.

Combinations of the four categories, in the mathematical sense of function composition, allow the expression of any operation on layers. Section 5 shows how objects, operations, and relationships are integrated into one model.

\section{Combining Objects and Fields - Example of usage}

The purpose of an integrated geographic data model in an open GIS environment is expressing the semantics of a geographic database in a way understandable and adaptable by any other model supported by autonomous and remote databases. Any portion of applications running at remote systems must be expressed without ambiguities in an intermediate model, GDM in this case, and vice versa (Figure 2).

GDM provides a formal syntax for describing the five constructs of relations, layers, virtual layers, object classes and constraints as well as the use of the four basic operations among layers. It uses syntactic rules of an Object-Oriented specification described in (Hadzilacos and Tryfona, 1994). Providing a formality for the representation of applications aids capturing semantics and handling important aspects of real-world entities represented in an information systems (Sheth, 1995).

In this section we present a sample usage of the GDM to show how objects $(\$ 4.1)$, layers $(\$ 4.3)$ and spatial relationships $(\$ 4.2)$ can be combined to express portions of geographic information. Objects and layers are combined in an orthogonal way $(\$ 3)$, 
spatial constraints among objects are translated into spatial relationships, while relations among layers are obtained by using the layer algebra ( $\$ 4.3)$.

Consider the following scenario: An application "running" on remote system A "asks" for a portion of the cadastral application located in remote system B using the statements: "identify sites suitable for a new park. Good candidates must be: within $1.25 \mathrm{Km}$ but no closer that $250 \mathrm{~m}$ from "motor roads" (accessible but not noisy) and not located in regions designated as "industrial" or "residential".

The basic goal -except from transferring the data (maps, records, etc.)- is to distiguish and tranfer objects, relationships among them, space-varying attributes (i.e., fields, layers) and their semantics in an understandable way from one location to the other.

This application requires the creation of a zone at the specified distance from motorways which must be overlaid with sites that are not industrial or residential. The result is a (virtual) layer representing candidate sites for a park. The result is derived from the overlay of two layers: one representing accessible but not noisy sites, and one representing the land use of our area of interest.

The above requirements can be formally stated within GDM as follows:

- DEFINE LAYER 1 LANDUSE

ATTR (USAGE, STRING)

GEOMETRIC TYPE REGION

- DEFINE LAYER 2 ROADS

ATTR (ROAD_TYPE, STRING),

(WIDTH, REAL)

GEOMETRIC TYPE ARC

- DEFINE VIRTUAL_LAYER 3 AS COMPUTE SPATIAL

$(2$, BUFF_ZONE_ $\leq$ _1.25KM=BUFFER $(1.25$, ROAD_TYPE $=" M O T O R W A Y "))$

- DEFINE VIRTUAL_LAYER 4 AS COMPUTE SPATIAL

(2, BUFF_ZONE_ $\geq \_250 \mathrm{M}=\mathrm{BUFFER}(250$, ROAD_TYPE="MOTORWAY"))

- DEFINE VIRTUAL_LAYER 5 AS OVERLAY $(3,4)$

- DEFINE VIRTUAL_LAYER 6 AS COMPUTE ATTRS $(5, \mathrm{ZONE}=$

$\left\{\begin{array}{l}\text { true, if } \mathrm{BUFF} \text { _ZONE } \leq 1.25 \mathrm{Km} \wedge \text { A BUFF_Z } \mathrm{BONE} \succ 250 \mathrm{~m}=\text { true } \\ \text { false }\end{array}\right\}$ )

- DEFINE VIRTUAL_LAYER 7 AS COMPUTE ATTRS $(1$, CANDIDATE_SITES $=$

$\left\{\begin{array}{l}\text { true, if USAGE } \neq \text { "Industrial" ^ USAGE } \neq \text { " Residential" } \\ \text { false, otherwise }\end{array}\right\}$

- DEFINE VIRTUAL_LAYER 8 AS RECLASS OF (7, CANDIDATE_SITES)

- DEFINE VIRTUAL_LAYER 9 AS OVERLAY $(6,8)$

- DEFINE VIRTUAL_LAYER 10 AS COMPUTE ATTRS

$\left(9, \mathrm{PARK} \_\right.$SITES $\left.=\left\{\begin{array}{l}\text { true }, \text { if } \mathrm{CANDIDATE} \text { _SITES }=\text { true } \wedge \mathrm{ZONE}=\text { true } \\ \text { false } \text { otherwise }\end{array}\right\}\right)$

- DEFINE OBJECT CLASS PARK_SITE ON LAYER 10 
Since remote systems "understand" GDM they can exchange objects, properties, layers, operations on layers and spatial integrity constraints.

\section{Conclusions and Future Work}

Interoperability among information systems is a major research focus for various domain areas. All approaches used so far assume the existence of a generic data model known to all cooperating remote systems.

In this paper we addressed the issue of information modeling for interoperable geographic database systems. Firstly, we discussed the particular semantics of spatial data whose representation and interchange are critical in an open environment. Next, we described a Geographic Data Model for exchanging spatial data, operations and these semantics among remote systems. GDM provides mechanisms to represent geographic object classes, layers (fields), operations on them, and spatial constraints. It is proposed as the intermediate, enterprise database model understandable by all remote systems comprising the interoperating environment.

Using a generic model as the intermediate step eliminates inconsistencies and redundancies during the phase of exchanging modeled information among systems. Remote systems must therefore (a) understand the domain specific semantics encapsulated by this model, and (b) provide mechanisms for translation from their model to GDM and vice versa. Flexibility and openness are not violated however, since each remote system may use its own model and facilities locally. GDM is used only when communicating with dissimilar systems.

A concept-to-concept mapping and analogies between various spatial data models and our proposed model are critical. Additionally, providing mechanisms for lossless transformation from existing spatial data models to the GDM are required. Prototypes built for different domain areas are also needed in order to understand their special or additional needs.

\section{Acknowledgments}

We would like to thank Thanasis Hadzilacos and Max. J. Egenhofer for their fruitful discussions and the Geographic Database Management Group of the Department of Spatial Information Science and Engineering for suggestions on earlier versions of this work.

\section{References}

1. Abel, D. J., and Kilby, P. J., 1994, The Systems Integration Problem. International Journal of Geographical Information Systems, vol. 8, no. 1, pp. 1-12.

2. Camara, G., Feritas, U.M., Souza, R.C.M., and Casanova, M.A., 1992, SPRING: Procassemanto de Imagens e Dados Georefenciados. Proceedings of $\mathrm{V}$ Brazilian Symposium on Computer Graphics and Image Processing (SIBGRAPI 92), pp. 233242 .

3. Cohen, D., Larson, G., and Berke, L., 1993, Role of Interoperability in Business Application Development. Proceedings of the ACM SIGMOD, 1993. 
4. Couclelis, H., 1992, People Manipulate Objects (but cultivate fields): Beyond the Raster-Vector Debate in GIS. Lecture Notes in Computer Science, Springer-Verlag (639), Proceedings of the International Conference GIS: From Space to Territory: Theories and Methods of Spatio-Temporal Reasoning in Geographic Space, Pisa, Italy, September 1992.

5. Delis, V., Hadzilacos Th., and Tryfona N., 1994, An Introduction to Layer Algebra. Advances in GIS Research, Proceedings of the 6th International Symposium on Spatial Data Handling, Edinburgh, UK, September 1994.

6. DeLorenzi, A., Wolf, A., 1993, A Protocol for Cooperative Spatial Information Managers. Proceedings of DBTA Workshop on Interoperability of Database Systems and Database Applications, Fribourg, Switzerland, 1993.

7. Egenhofer, M., and Herring, J., 1991, Categorizing Binary Topological Relationships Between Regions, Lines and Points in Geographic Databases. Technical Report, Orono, ME: Department of Surveying Engineering, University of Maine.

8. Elmagarmid A. and Pu, C. (Eds), 1990, Special Issue on Heterogeneous Databases, ACM Computing Surveys, 22, 3.

9. Goh, C., H., Madnick, S., and Siegel. M., 1994, Context Interchange: Overcoming the Challenges of Large Scale Interoperable Database Systems in a Dynamic Environment. Proceedings of the Third International Conference on Information and Knowledge Management (CIKM), Maryland, 1994.

10. Hadzilacos Th., 1995, Personnal Communication.

11. Hadzilacos Th., and Tryfona N., 1992, A Model for Expressing Topological Integrity Constraints in Geographic Databases. Lecture Notes in Computer Science, Springer-Verlag (639), Proceedings of the International Conference GIS: From Space to Territory: Theories and Methods of Spatio-Temporal Reasoning in Geographic Space, Pisa, Italy, September 1992.

12. Hadzilacos Th., and Tryfona N., 1994, Logical Data Modeling for Geographic Databases. International Journal of Geographic Information Systems, $(9,6)$.Kim, W., 1995, "Modern Database Systems: The Object-Oriented Model, Interoperability and Beyond", ACM Press.

13. Kashyap, V., and Sheth, A., 1994, Semantics-based Information Brokering. Proceedings of the Third International Conference on Information and Knowledge Management (CIKM), Gaithensburg, MD, November, 1994.

14. Mannal, D., and Burgara, K., 1993, Enhancing Inter-operability and Data Sharing in Medical Informaion Systems. Proceedings of the ACM SIGMOD, 1993.

15. Manola, F. , Heiler, S., Georgakopoulos, D., Hornick, M., and Brodie, M., 1992, Distributed Object Management. Intermational Journal of Intelligent Cooperative Information Systems, $1,1$.

16. Pascoe, R. T. and Penny, J. P., 1995, Constructing Interfaces between (and within). Geographical Information Systems, vol. 9., no. 3, pp. 275-291.

17. Sciore, E., Siegel, M., and Rosenthal, A., 1994, Using Semantic Values to Facilitate Interoperability Among Heterogeneous Information Systems. ACM Transactions on Database Systems, vol. 19, no. 2. 
18. Schek, H.J., and Wolf, A., 1992, Cooperation Between Autonomous Operation Services and Object Database Systems in a Heterogeneous Environment. Proceedings of the IFIP TC2/WG2.6 Conference in semantics of Interoperable DatabaseSystems, Victoria, Australia, November 1992.

19. Sheth, A., 1995, Data Semantics: what, where and how?. Technical Report TR-CS95-003, LSDIS Lab, Dept. of CS, Univ. of GA, September 1995. To appear in "Database Application Semantics," Proceedings of the 6th IFIP Working Conference on Data Semantics (DS-6), R. Meersman and L. Mark (Eds.), Chapman abd Hall, London, UK, 1996.

20. Sheth, A., and Karabaitis, G., 1993, Multidatabase Interdependencies in Industry. Proceedings of the ACM SIGMOD, 1993.

21. Sheth and Kalinichenko, 1992, Information Modeling in Multidatabase Systems: Beyond Data Modeling. Proceedings of the First International Conference on Information and Knowledge Management (CIKM), Baltimore, MD, November, 1994.

22. Soley, R.M., 1992, Using Object Technology to Integrate distributed applications. In Enterprise Integration Modeling, Proceedings of the First International Conference, MIT Press, Cambridge, Mass, 446-454.

23. Tryfona N., and Hadzilacos Th., 1995, Geographic Applications Development: Models and Tools for the Conceptual Level. Proceedings of the ACMGIS'95 Workshop, Baltimore, MD.

24. Tryfona, N., 1994, A Structured Database Methodology for Geographic Applications. Ph.D. Thesis, Dept. of Computer Engineering and Informatics, University of Patras, Greece.

25. Voisard, A., and Schweppe, H., 1994, A Multilayer Approach to the Open GIS Design Problem. Proceedings of the ACM GIS' 94 Conference.

26. UtilNets, 1994, Work Program of Brite-Euram Project 7120, GD XII, European Union, Brussels, Belgium. 